\title{
ION IMPLANTED PIEZORESISTIVE CANTILEVER DESIGN AND PERFORMANCE
}

\author{
S.-J. Park ${ }^{l}$, A.J. Rastegar ${ }^{l}$, J.R. Mallon Jr. ${ }^{2}$, A.A. Barlian ${ }^{l}$, T.H. Fung ${ }^{2}$ and B.L. Pruitt ${ }^{l}$ \\ ${ }^{1}$ Department of Mechanical Engineering, Stanford University, Stanford, California, USA \\ ${ }^{2}$ Department of Electrical Engineering, Stanford University, Stanford, California, USA
}

\begin{abstract}
We present design guidelines for ion implanted piezoresistive cantilevers. Most analytical models assume cantilevers have uniform doping profiles, whereas ion implanted piezoresistors with graded doping profiles are commonly fabricated in commercial force and pressure sensors. To address this issue, we modified existing models to predict the performance of ion implanted piezoresistors. Experiment, simulation, and our analytical model of force sensitivity and force resolution agree with less than $20 \%$ error. Using the analytical model, we also demonstrate a method to choose design and operating parameters to optimize performance.
\end{abstract}

\section{INTRODUCTION}

MEMS piezoresistive microcantilevers have become increasingly popular as force and displacement sensors since the first piezoresistive AFM cantilevers by Tortonese [1]. Piezoresistors have advantages such as large dynamic range, large deflection, relatively small size, simple fabrication, and straightforward signal-conditioning circuitry. Piezoresistive cantilevers are used where other transduction methods are impractical [2] and Harley and Kenny's [3] analyses of noise and sensitivity are widely used in maximizing sensitivity for limited power. However, piezoresistor design remains challenging due to the many coupled parameters such as cantilever dimensions (width, length, and thickness), piezoresistor dimensions, fabrication process parameters (doping concentration, annealing time and temperature), and bias voltage which must be chosen carefully to optimize performance (bandwidth, stiffness, resolution, power dissipation and nonlinearity). For example, increasing sensitivity by reducing piezoresistor doping and increasing bias voltage increases power dissipation and noise. Harley [3] first showed a method to design piezoresistive cantilevers, but only for epitaxial piezoresistors with uniform doping profiles [4]. However, ion-implanted piezoresistive sensors with non-uniform doping profiles are commonly specified for their low cost and simple fabrication. Here, we extend analytical model to predict sensitivity and resolution of piezoresistive cantilevers with arbitrary doping profiles. We validate the model with simulation and experimental measurements. We also show how to choose optimal design parameters that satisfy the complex parameter interaction of piezoresistive cantilevers.

\section{ANALYICAL MODEL}

To calculate sensitivity and resolution of piezoresistive cantilevers with an arbitrary dopant profile, we should consider two profiles: stress distribution and doping concentration profile. Stress is distributed across the length and thickness of a cantilever as per Euler's beam equation; and doping concentration varies in the thickness of cantilever for common doping techniques such as epitaxial growth and ion-implantation. Therefore, we model the piezoresistor in depth as a composite of many thin slices of piezoresistors connected in parallel, with the change in resistance in each slice depending on the stress and piezoresistive coefficient for that slice. By integrating the change in resistance in thin slices of a piezoresistor, we can compute force sensitivity in terms of resistance change $(\Delta R / R)$, cantilever dimension (length, $l$, width, $w$, thickness, $t)$, piezoresistor length $\left(l_{p}\right)$, maximum longitudinal piezoresistivity as a function of direction at $300^{\circ} \mathrm{K}\left(\pi_{l_{-} \max }\right)$, electronic charge $(q)$, mobility $(\mu)$, doping concentration $(p)$, piezoresistance factor $(P)$, distance from neutral axis $(z)$ :

$$
S_{F \Omega}=\frac{\frac{\Delta R}{R}}{F}=\frac{12\left(l-\frac{1}{2} l_{p}\right) \pi_{l_{-} \max }}{w t^{3}} \frac{\int_{-t / 2}^{t / 2} q \mu p P z d z}{\int_{-t / 2}^{t / 2} q \mu p d z}[\Omega],
$$

We define an efficiency factor, $\beta^{*}$, by dividing force sensitivity by the theoretical maximum force sensitivity which can be achieved if the dopant atoms exist only at the surface $(z=t / 2$, Fig. 1) and the doping concentration is small enough to maintain the maximum piezoresistance factor $(P=1)$ :

$$
\beta^{*}=\frac{2}{t} \frac{\int_{-t / 2}^{t / 2} q \mu p P z d z}{\int_{-t / 2}^{t / 2} q \mu p d z} .
$$

$\beta^{*}$ is function only of cantilever thickness and process parameters, so the $\beta^{*}$ of cantilevers from the same wafer is constant and independent of cantilever length and width. $\beta^{*}$ differs from the efficiency factor $(\beta)$ defined by Tortonese and Harley [3]. $\beta^{*}$ includes the piezoresistance factor $(P)$ inside the integral of the numerator only. While $\beta$ represents the conductivity and piezoresistivity weighted average of stress, $\beta^{*}$ is the conductivity weighted average of the product of piezoresistivity and stress. $\beta$ neglects the depth-weighted, piezoresistivity effect on force sensitivity because the piezoresistance factors in the numerator and denominator cancel, as in the case of epitaxy. Because $\beta^{*}$ is a ratio of the depth-weighted and maximum force sensitivities, we can now calculate sensitivity by multiplying $\beta^{*}$ and maximum force sensitivity. This extended model predicts the sensitivity of ion-implanted cantilevers, where the piezoresistance factor rapidly varies with depth.

Using $\beta^{*}$ and considering a $1 / 4$-active Wheatstone bridge

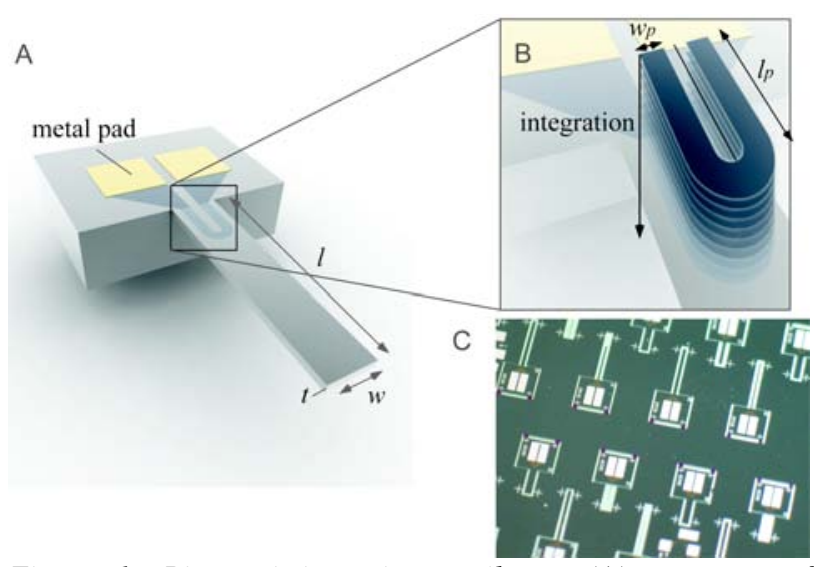

Figure 1: Piezoresistive microcantilever: (A) geometry of cantilever, $(B)$ geometry of piezoresistor. We can compute force sensitivity by integrating the change in resistance in thin slices of a piezoresistor. (C) custom made piezoresistive cantilevers. 

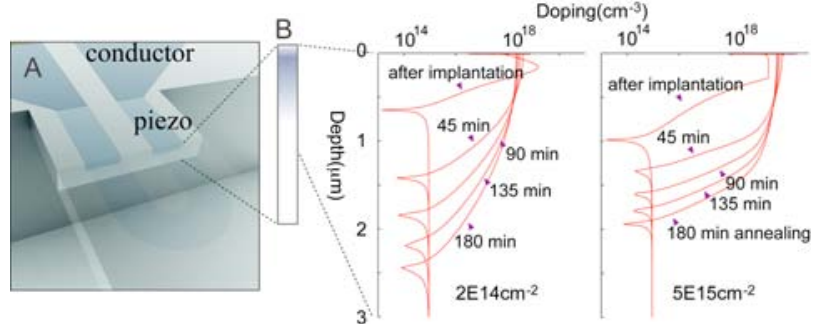

Figure 2: TSUPREM4 simulation of doping concentration profile of cantilever every $45 \mathrm{~min}$ annealing. Inert $\mathrm{N}_{2}$ anneal at $1100{ }^{\circ} \mathrm{C}$ for low dose $\left(2 \mathrm{E} 14 \mathrm{~cm}^{-2}\right.$, left $)$, and at $1000{ }^{\circ} \mathrm{C}$ for high dose $\left(5 E 15 \mathrm{~cm}^{-2}\right.$, right $)$.

configuration (voltage output, $V_{\text {out }} \approx V_{\text {bias }} / 4 \cdot \Delta R / R$ ), force sensitivity may be expressed in terms of voltage as,

$$
S_{F V}=\frac{3\left(l-\frac{1}{2} l_{p}\right) \pi_{l_{-} \max }}{2 w t^{2}} \beta * \gamma V_{\text {bias }},
$$

where $V_{\text {bias }}$ is bias voltage and $\gamma$ is a geometric factor defined as the ratio of the resistance of the strained region in the piezoresistor and total resistance including unstrained regions, interconnects, and contact pads (Fig. 1). Because these regions do not change resistance with applied force, force and displacement sensitivity are decreased.

Noise and force sensitivity determine force resolution:

$$
F_{\min }=\frac{\sqrt{\frac{\alpha V_{\text {bias }}^{2}}{2 l_{p} w_{p} N_{z}} \ln \left(\frac{f_{\max }}{f_{\min }}\right)+8 k_{B} T R_{s} \frac{l_{p}}{w_{p}}\left(f_{\max }-f_{\min }\right)}}{\frac{3\left(l-\frac{1}{2} l_{p}\right) \pi_{l_{-} \max }}{2 w t^{2}} \beta^{*} \gamma V_{\text {bias }}},
$$

where $\alpha$ is the Hooge noise parameter [5], $w_{p}$ is piezoresistor width, $N_{z}$ is the integration of doping concentration across thickness, $k_{B}$ is Boltzmann's constant, $T$ is operating temperature, $R_{s}$ is sheet resistance, $f_{\max }$ and $f_{\min }$ are respectively the maximum and minimum frequency. The first term in the numerator is $1 / f$ noise and the second term is Johnson noise of the U-shaped piezoresistor.

To predict force resolution of a cantilever with an arbitrary doping profile, we first calculate $\beta^{*}$ (Eq. 2) using the doping profile simulated by TSUPREM4 (Fig. 2). We then compute the predicted sensitivity by multiplying $\beta^{*}$ and the maximum sensitivity (Eq. 3).
Force resolution is calculated by dividing noise by the sensitivity (Eq. 4).

\section{VALIDATION}

We validated the analytical model by comparing it with numerical simulation and experimental measurements of custom fabricated, ion-implanted cantilevers. We designed and fabricated ion-implanted piezoresistive cantilevers with varied dimensions $(l$ : 1.7 to $6 \mathrm{~mm} ; w$ : 30 to $400 \mu \mathrm{m}, t: 7$ to $50 \mu \mathrm{m}, l_{p}: 200$ to $350 \mu \mathrm{m}, w_{p}$ : 10 to $20 \mu \mathrm{m})$ and varied fabrication process conditions (implantation energy: $50 \mathrm{keV}$, dose: $5 \mathrm{E} 15$ to $5 \mathrm{E} 16 \mathrm{~cm}^{-2}$, annealing temperature: 1000 to $1150^{\circ} \mathrm{C}$, oxidation time: 15 to $45 \mathrm{~min}, \mathrm{~N}_{2}$ annealing time: 5 to $35 \mathrm{~min}$ ) as described previously $[2,6]$. We measured the effective spring constants, force sensitivities, and first-mode resonant frequencies of cantilevers experimentally using a Laser Doppler Vibrometer (Polytec OFV3001) and resonance techniques [6,7]. In addition, we computed resistance change by integrating the stress and stress-induced resistivity change of each piezoresistor element for a force applied at the tip of cantilever using a commercial finite element method code, COMSOL. We calculated $\beta^{*}$, force sensitivity and force resolution using our analytical model (Eq. 2, 3, and 4) based on two different piezoresistance factors: Harley's empirical model [3] and Kanda's theoretical values [8] (Fig. 3A).

The analytical model agrees with experimental data $(<20 \%$ error, Fig. 3) and the numerical solution ( $<5 \%$ error). Fig. $3 \mathrm{~A}$ shows the changes in resistance based on the analytical model and experimental results when a point force is applied at the tip. Our experimental data are closer to the analytical prediction using Harley's piezoresistance factor rather than Kanda's (Fig. 3A). Fig. 3B and Fig. 3C show $\beta^{*}$ and force sensitivity, respectively, as a function of diffusion length for a $15 \mu \mathrm{m}$ thick piezoresistive cantilever. The predicted $\beta^{*}$ and force sensitivity of cantilevers with various dimension and process conditions are in good agreement with experimental results. As expected, $\beta^{*}$ of high dose cantilevers with long diffusion lengths significantly decreases with increasing diffusion length, because diffusion causes the dopant atoms to move away from the top surface (highest stress region) resulting in decreased $\beta^{*}$ and force sensitivity. In addition, $\beta^{*}$ and force sensitivity of high dose, short diffusion length cantilevers are independent of dose because of the solid solubility limit. We also found that $\beta^{*}$ and force sensitivity of low dose cantilevers increase with annealing because the piezoresistance factor effect more than
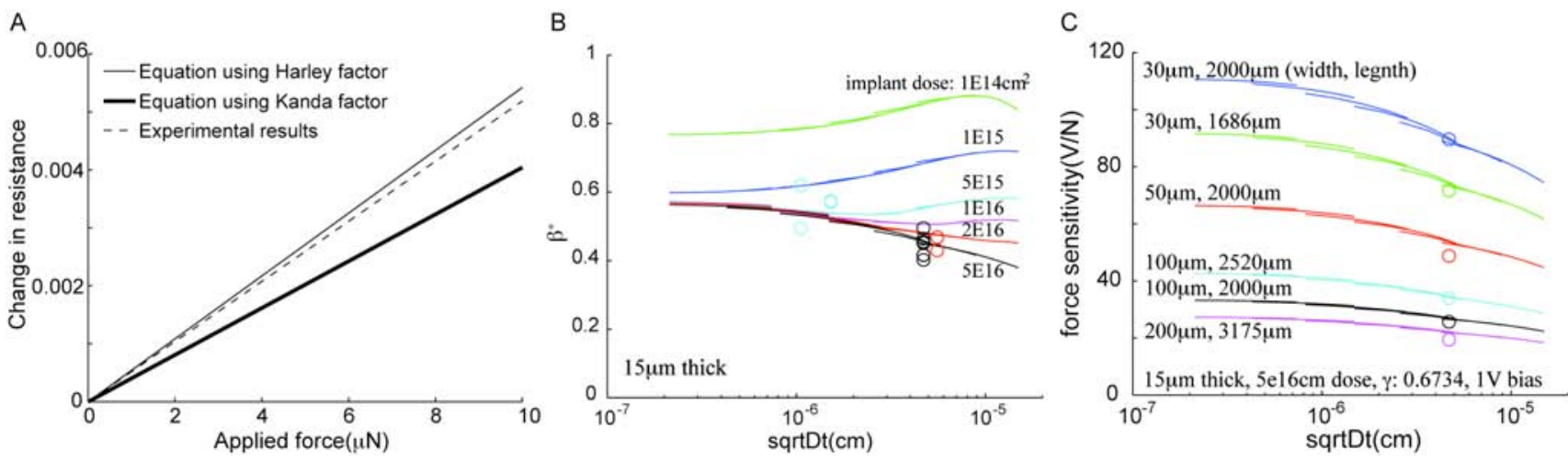

Figure 3: Validation of the analytical model: (A) Comparison of analytical model and experimental results of changes in resistance of a typical piezoresistive cantilever (2000 $\mu \mathrm{m}$ long, $30 \mu \mathrm{m}$ wide, $15 \mu \mathrm{m}$ thick cantilever with $350 \mu \mathrm{m}$ long by $10 \mu \mathrm{m}$ wide U-shaped piezoresistor) based on piezoresistance factors of Harley's empirical model [3] and Kanda's theoretical values, (B) $\beta^{*}$ of $15 \mu \mathrm{m}$ thick piezoresistive cantilever, and $(C)$ force sensitivity of piezoresistive cantilevers with various cantilever dimensions. The analytical model (solid lines) is in good agreement with experimental results (circles). 
compensates for depth effects (lower doping concentrations have higher piezoresistance factors, while bending stress decreases linearly with depth).

\section{OPTIMIZATION}

We present four parameter categories for optimizing piezoresistive cantilever performance: 1) cantilever dimension (width, length, and thickness), 2) piezoresistor dimension, 3) bias voltage, and 4) fabrication process parameters (doping concentration, annealing time and temperature).

\section{Cantilever Dimensions}

Measurement bandwidth, desired stiffness of cantilever and desired cantilever thickness determine cantilever length and width. The maximum bandwidth is usually limited by the resonant frequency $\left(f_{n}\right)$ of the cantilever. The stiffness $\left(k_{c}\right)$ of cantilever is set by the sample stiffness: a soft cantilever is good for a soft sample because stiff cantilevers can damage soft samples. The minimum useful cantilever thickness is limited by selected process parameters, such as ion implant energy, dose, anneal temperature and time.

The desired bandwidth, stiffness and cantilever thickness determine the optimal cantilever length and width:

$$
\begin{aligned}
& l=\left(\frac{E}{\rho_{s}}\right)^{1 / 4}\left(\frac{t}{2 \pi f_{n}}\right)^{1 / 2} \\
& w=\frac{4 k}{E}\left(\frac{l}{t}\right)^{3}
\end{aligned}
$$

where $E$ is Young's modulus and $\rho_{s}$ is density of the beam. Fig. 4 suggests lengths of silicon cantilevers over a range of resonant frequency and thickness.

\section{Piezoresistor Dimensions}

We choose piezoresistor length and width to minimize force resolution. Force resolution is inversely proportional to the piezoresistor width (Eq. 4). Therefore, we select a piezoresistor width as large as possible $\left(w_{\mathrm{p}}=w / 2\right)$. However, the choice of piezoresistor length is not simple: while a longer piezoresistor is better for $1 / f$ noise, a shorter piezoresistor is better for Johnson noise and force sensitivity (Eq. 4). We find the optimal ratio of cantilever and piezoresistor length $\left(a=l_{p} / l\right)$ by differentiating the force resolution with respect to $a$. The optimal $a$ is function of the ratio of $1 / f$ noise and Johnson noise, integrated over the bandwidth, when piezoresistor length extends the full cantilever length (Fig. $5)$ :

$$
\sqrt{\frac{a^{3}+2 a^{2}}{2-3 a}}=\left(\frac{1 / f \text { noise }(\mathrm{V})}{\text { Johnson noise }(\mathrm{V})}\right)_{l_{p=l}} .
$$

The optimal $a$ is $2 / 3$ for $1 / f$ noise dominant cantilevers and 0 for Johnson noise dominant cantilevers. Thus, the optimal piezoresistor length can be between 0 and $2 / 3$ of cantilever length depending on the ratio of $1 / \mathrm{f}$ noise and Johnson noise. Piezoresistor thickness is subsequently determined once we select the fabrication process.

\section{Bias Voltage}

Once the cantilever and piezoresistor dimensions are determined from Eq. 5, 6 and 7, we choose a bias voltage by considering power dissipation of the piezoresistor. Although higher bias voltage has little effect on the force resolution of a $1 / f$ noise dominant cantilever, it significantly improves force resolution of Johnson noise dominant cantilevers (Eq. 4). However, high bias voltage will increase power dissipation and operating temperature,

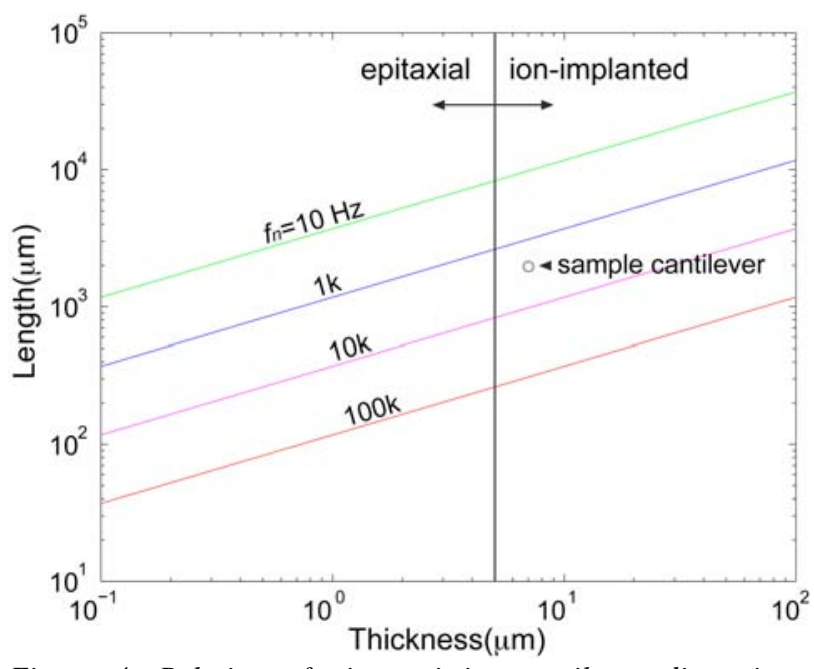

Figure 4: Relation of piezoresistive cantilever dimensions. Target resonant frequency (bandwidth) determines cantilever dimensions. The circle (2000 $\mu \mathrm{m}$ long and $7 \mu \mathrm{m}$ thick, and $2.5 \mathrm{kHz}$ resonant frequency) is one of our sample cantilevers.

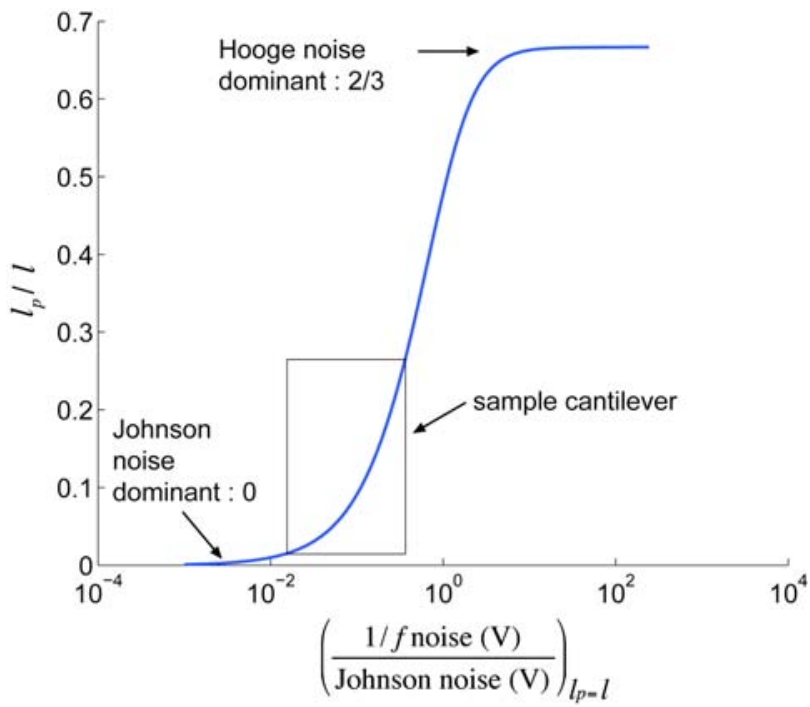

Figure 5: Optimization of piezoresistor length. The optimal piezoresistor length can be determined by the ratio of $1 / f$ noise and Johnson noise when piezoresistor extends the full cantilever. We found that the optimal length ratio of $1 / f$ noise dominants and Johnson noise dominant cantilever is $2 / 3$ and 0 respectively. The box indicates optimal piezoresistor length range of a typical cantilever.

and could lead to mechanical destruction or piezoresistance reduction (piezoresistivity is inversely proportional to temperature). Therefore, we should choose as high a bias voltage as power dissipation will allow.

\section{Fabrication Process Parameters}

Once cantilever and piezoresistor dimensions and bias voltage are set, then we can calculate force resolution for a variety of process conditions using Eq. 2, 3, and 4. Fig. 6 shows force resolution performance of a sample cantilever over a range of process conditions (dose and diffusion length). We can choose desired fabrication process parameters such as doping concentration, annealing time and temperature from Fig. 6. 


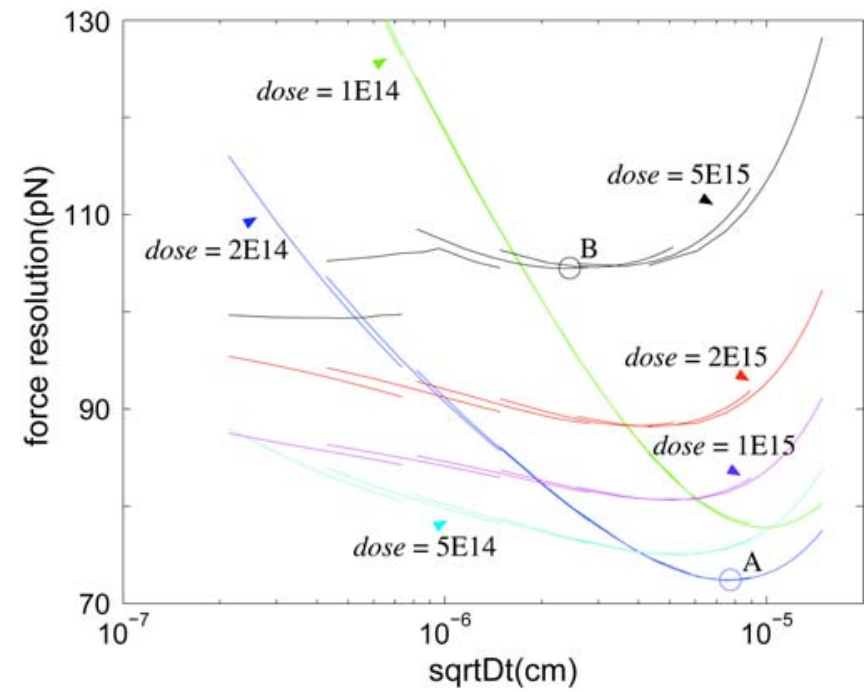

Figure 6: Optimization of typical piezoresistive cantilevers. Target design conditions are $2.5 \mathrm{kHz}$ bandwidth, $0.05 \mathrm{~N} / \mathrm{m}$ stiffness, <10 $m W$ power, $<5 \mathrm{~V}$ bias voltage, and $700 \Omega$ resistance. Circle $A$ and $B$ indicates the lowest force resolution of cantilevers with low dose and high dose respectively. The design parameters and specification of these cantilevers are summarized in Table 1.

\section{Sample Piezoresistive Cantilever Optimization}

We chose design and operating parameters to optimize a sample piezoresistive cantilever for use with an INA 103, Texas Instruments, USA, a low Johnson noise commercial instrumentation amplifier. The desired design conditions included 1 to $2500 \mathrm{~Hz}$ bandwidth, $0.05 \mathrm{~N} / \mathrm{m}$ stiffness, <10 $\mathrm{mW}$ power dissipation, $<5 \mathrm{~V}$ bias voltage, $>700 \Omega$ resistance, and a $7 \mu \mathrm{m}$ thick silicon on insulator (SOI) wafer. From Eq. 5 and 6, the cantilever should be $2000 \mu \mathrm{m}$ long and $30 \mu \mathrm{m}$ wide (circle in Fig.4). Based on TSUPREM4 simulation data for a variety of ion implantation and annealing conditions (1E14 to $5 \mathrm{E} 16 \mathrm{~cm}^{-2}$ dose, 900 to $1150{ }^{\circ} \mathrm{C}$ and 0 to 180 minutes anneals), we calculated $a$, bias voltage, $\beta^{*}$, force sensitivity, noise and force resolution from Eq. 2, 3, 4, 7 (Fig. 5 box and Fig. 6 circles). The parameters are summarized in Table 1 for low vs. high dose cases.

\section{CONCLUSION}

We have presented an analytical model to predict force sensitivity and resolution of ion implanted piezoresistive microcantilevers. We characterized several piezoresistive cantilevers over a range of process conditions and verified that the model agrees with simulation and experiment with less than $20 \%$ error. We have also shown this model can be used for optimization of cantilever performance for a given process. This optimization technique is useful in the design of piezoresistive sensors for a variety of applications with complex design conditions, e.g. broad bandwidth $(>100 \mathrm{kHz})$, small force resolution $(\sim \mathrm{pN})$, large dynamic range $(\mathrm{nN} \sim \mathrm{mN})$, or CMOS compatible sensors.

\section{ACKNOWLEDGEMEMTS}

This work was performed in part at the Stanford Nanofabrication Facility (a member of the National Nanotechnology Infrastructure Network) which is supported by the National Science Founding under Grant 9731293, its lab members, and the industrial members of the Stanford Center for Integrated Systems. Funding was also provided under NSF ECCS-0449400,
Table 1: Design and operating conditions and specification of piezoresistive cantilevers with low dose (circle A in Fig. 6) and high dose (circle B in Fig. 6).

\begin{tabular}{lcc}
\hline \hline Parameters & $\begin{array}{c}\text { Low Dose } \\
\left(2 \mathrm{E} 14 \mathrm{~cm}^{-2}\right)\end{array}$ & $\begin{array}{c}\text { High Dose } \\
\left(5 \mathrm{E} 15 \mathrm{~cm}^{-2}\right)\end{array}$ \\
\hline$l w t(\mu \mathrm{m})$ & $2000 \times 30 \times 7$ & $2000 \times 30 \times 7$ \\
$l_{p} w_{p} t_{p}(\mu \mathrm{m})$ & $80 \times 15 \times 2.18$ & $220 \times 15 \times 1.79$ \\
anneal temperature $\left({ }^{\circ} \mathrm{C}\right)$ & 1100 & 1000 \\
anneal time $(\mathrm{min})$ & 135 & 135 \\
bias voltage $(\mathrm{V})$ & 5 & 2.6 \\
Specification & & \\
\hline resistance $(\Omega)$ & 2500 & 700 \\
resonant frequency $(\mathrm{kHz})$ & 2.5 & 2.5 \\
stiffness $(\mathrm{N} / \mathrm{m})$ & 0.05 & 0.05 \\
power dissipation $(\mathrm{mW})$ & 10 & 10 \\
$\beta^{*}$ & 0.68 & 0.44 \\
force sensitivity $(\mathrm{V} / \mathrm{N})$ & 4910 & 1630 \\
noise $(\mathrm{nV})$ & 356 & 170 \\
force resolution $(\mathrm{pN})$ & 72 & 104 \\
\hline \hline
\end{tabular}

ECCS-0425914, and NIH 1 R01 EB006745-01A1. S-JP was supported by a Samsung fellowship.

\section{REFERENCES}

[1] M. Tortonese, R. C. Barrett, and C. F. Quate, "Atomic Resolution with an Atomic Force Microscope using Piezoresistive Detection,” Appl. Phys. Lett., 62, 834 (1993).

[2] S.-J. Park, M. B. Goodman, and B. L. Pruitt, "Analysis of Nematode Mechanics by Piezoresistive Displacement Clamp,” Proc. Natl. Acad. Sci. USA, 104, 17376 (2007).

[3] J. A. Harley and T. W. Kenny, "1/F Noise Considerations for the Design and Process Optimization of Piezoresistive Cantilevers," J. Microelectromech. Sys, 9, 226 (2000).।

[4] J. A. Harley and T. W. Kenny, "High-sensitivity Piezoresistive Cantilevers under 1000A thick," Appl. Phys. Lett., 75, 289 (1999).

[5] F.N. Hooge, "1/f Noise Sources," IEEE Trans. Electron Devices, 41, 1926 (1994).

[6] B. L. Pruitt and T. W. Kenny, "Piezoresistive Cantilevers and Measurement System for Characterizing Low Force Electrical Contacts," Sens. Actuators A, 104, 68 (2003).

[7] B. L. Pruitt, W. T. Park, and T. W. Kenny, "Measurement System for Low Force and Small Displacement Contacts," J. Microelectromech. Sys, 13, 220 (2004).

[8] Y. Kanda, "Piezoresistance Effect of Silicon," Sens. Actuators A, 28, 83 (1991). 